\title{
The common oro-dental problems among patients attending Ohud dental care and implant center at Bogra, Bangladesh
}

\author{
Hoque $\mathrm{S}^{1^{*}}$, Jahan $\mathrm{I}^{2}$ \\ ${ }^{1}$ Ohud dental care and implant center, South Thanthania, Bogra, Bangladesh ${ }^{2}$ Atish Dipankar University of \\ Science and Technology, Dhaka, Bangladesh
}

[Received: February 20, 2015; Accepted: May 29, 2015]

\begin{abstract}
A descriptive type of cross sectional study on exploring the common oro-dental problems among the patients attending outpatient department of Ohud Dental Care and implant center, Bogra was conducted during the period from May to July' 2013. The objectives of this study were to identify the common oro-dental problems for assessing the level of knowledge regarding dental care practices among the respondents. A questionnaire based data were collected and the sample compares 414 respondents of all age groups. The highest percentage of the patients had complaints of periodontitis $(73.9 \%)$ followed by gingivitis $(64.3 \%)$, dental caries $(40.6 \%)$, attrition $(10.4 \%)$, mobility $(5.8 \%)$, broken teeth $(5.6 \%)$ and alveolar abscess $(6.3 \%)$. Considering the pattern of inflammation, $425.5 \%$ had inflammatory or-dental problems and $56.6 \%$ had non-inflammatory oro-dental problems. On examination also revealed that $31.9 \%$ unhealthy gums, $3.9 \%$ had unhealthy tonsils, $10.3 \%$ had diseased tongue, $10.7 \%$ had diseases mucosa and $4.1 \%$ had apthus ulcer. The proportion of illiterate patients were higher among the inflammatory disease $(16.7 \%)$ compared to non-inflammatory disease $(9.8 \%)$, where as respondents having graduate level of education were higher among the non- inflammatory disease $(30.8 \%)$ compared to inflammatory diseases $(8.3 \%)$. There was significant association between the oro-dental problems and food habit ( $>0.05$ ) except liking of sweet and habit of area nut and lime $(\mathrm{p}<0.05)$. This indicated that the proportion of non-inflammatory dental problems were higher among the patients habituate with sweet $(82.5 \%)$ compared to inflammatory disease $(72.2 \%)$. But the proportion of inflammatory disease was higher among the patients having habituated with chewing areca nut line $(35.0 \%)$ than non- inflammatory disease $(26.1 \%)$. Knowledge of causes of dental problems higher among the patients having non-inflammatory disease $(62.0 \%)$ compared to inflammatory disease (46.7\%) and the difference was statistically significant $(\mathrm{p}<0.001)$. Similarly, the proportion of visiting dental surgeon was significantly $(\mathrm{p}<0.001)$ higher among the patients having non-inflammatory disease $(4.4 \%)$.
\end{abstract}

Key words: Oro-dental problems, out patients, Bogra, Bangladesh

\section{INTRODUCTION}

Dental problems are a public health problem in Bangladesh. Hence, the outline of these dental diseases needs to be identified by valid studies to assess the actual distribution of the problem in the community. Due to deficient of awareness of the people the dental diseases are increasing day by day. So it is the crying need of the country to have adequate information on pattern of dental diseases and to take necessary prevention program to fight against the dental diseases. Seeing as both the initiation and the continuation of our major problems in dental practice, dental diseases are in part nutrient and food in origin, it is logical and necessary that our management should be regarded to deal with these causes. Research has proved that partial inhibition or arrest of this disease can" be attained directly with food factor. For the last 30 or more years our primary task has been restarting, fabricating and rehabilitating teeth and mouths. Because of the increase in population, and the corresponding rise in dental needs, we appreciate more and more each day that we are fighting a losing battle by mere emphasis on therapy; this is obviously neither adequate nor progressive. Prevention not therapy is today's challenge and hopefully tomorrow's achievement [1], Multiple factors which are responsible for the causation of Dental diseases, but dietary factors especially consumption of fermentable carbohydrate plays a major role for the causation of dental diseases. Other factors like oral microbial enzymes, physical and chemical structure of teeth, poor oral hygiene, calcium and vitamin D deficiency in the diet, low fluoride content in drinking water are responsible for dental disease formation. Oro-dental hygiene is the most common accompaniment of dental diseases with other associated factors". WHO suggested three things for better oral health, includes cleaning mouth preferably after each meat, Use fluoride in drinking water or tooth paste and Eat less sugar [2]. Even dental caries is chronic progressive disease and not a life endangering disease, but it can sometimes be quite troublesome and is one of the most common problems in our country. The highest prevalence of dental disease among the school children and young adults is probably due to inadequate knowledge, ignorance about practice of dental hygiene, etiology, prevention and complication of the disease [3]. A set of sound healthy teeth is a valuable asset, it "contributes to good personal appearance in addition to be an efficient chewing apparatus. The slogan of the world health day of 1994 was "Oral health for healthy life" [4]. There is a strong association between dental diseases and practice of oral hygiene. Those who maintain their oral hygiene, they develop least dental diseases. The prevention of oro-dental diseases can 
be ensured by regular cleaning of the mouth and teeth. This tasks essentially an individual action and responsibility. But in case of child, it would not be possible to do this task alone and would need support from their parents and superiors [5]. Consequently the ultimate target of the researcher is to identify the common oro-dental problems and to assess the level of knowledge regarding dental care practices among the respondents. Generated information from this study may be of immense benefit for the policy planners of the country to implement necessary intervention to fight against the common oro-dental problems of the country.

\section{MATERIALS AND METHODS}

\section{Study area and population}

This study was carried out among outpatient department of Ohud dental care and implant center at Bogra district of Bangladesh. Information from all the patients with oro-dental problems attended to the outpatient department of Ohud dental care and implant center, Bogra for treatment were recorded accordingly. The study sample was free form mental disease, handicapped and avoid from seriously ill patients.

\section{Study design and duration of the study}

A descriptive type of cross sectional study on exploring the common oro-dental problems among the patients attending outpatient department of Ohud Dental Care and implant center in Bogra was conducted during the period from May to July' 2013 (3 months).

\section{Data Collection Instrument}

According to study objectives, all variables were enlisted and appropriate scales of measurements were determined. Accordingly questionnaires and examination form were developed. The instruments will tested on out patients department of Ohud dental care and implant center of similar characteristics and finalized.

\section{Data Collection Method}

Before data collection a request letter from the respective institute was handed over the appropriate authority area for taking permission and seeking assistance for smooth access data collection a fewnumbers of interviewers was selected and trained then for data collection technique. The interviewers were instructed to take informed consent from the prospective respondents before taking interview. Data collection process should be followed accordingly after the permission of authority the investigator collected data with the help of an interpreter. Interviewing and examining the patient collected data. The investigator asked the question in Bengali to the respondents and for the data it was written in English.

\section{Data Analysis}

All interviewed questionnaire was checked for its completeness and correctness before data entry, data was checked clean and edited properly before analysis. Questionnaire could be coded and codebook was prepared, data was entered into the computer by using statistical software namely excel and SPSS. In this study for data analysis frequency distribution, percentage, central tendency (mean) dispersion (standard deviation) and proportion of important variables will be calculated. The cross tabulation and association was determined chi-square $\left(\mathrm{x}^{2}\right)$ test for analyzing the relationship between independent variables and dependent variable; Correction was employed when data analysis in done in $2 \times 2$ tables. An alpha (a) level of 0.5 aws employed as a significance level for the entire statistical test.

\section{RESULTS}

\section{Socio-demographic characteristics}

The mean age of the respondents was $31.5 \pm 11.4$ years range from 15 to 79 years. Highest percentage of the patients had in the range of 25-34 years $(35.0 \%)$ followed by $15-24$ years $(29.7 \%)$ and lowest in the age range of 45 years and above $(15.5 \%)$. Out of 414 respondents, two third $(66.7 \%)$ were male and the rest one third $(33.3 \%)$ were female. More than one tenths $(12.8 \%)$ were illiterate, $22.5 \%$ had HSC level of education and $21.0 \%$ had degree and above On the contrary, more than one third of the respondent's fathers were illiterate (36.2\%). However, $24.2 \%$ had degree level of education. Regarding the mothers level of education, more than half $(54.1 \%)$ were illiterate followed by $16.7 \%$ had primary level of education, $11.6 \%$ had SSC and $8.7 \%$ had secondary level of education. Highest percentage of the father's were businessmen followed by service holder $(23.4 \%)$, daily laborer $(22.9 \%)$, and agricultural workers $(21.0 \%)$ Majority of the mothers were house wife $(91.1 \%)$ and $(8.2 \%)$ were service holders.

\section{Prototype of oro-dental problems}

It was observed that highest percentage of the patients had complaints of penodontitis (73.9\%) followed by gingivitis (64.3\%), dental caries $(40.6 \%)$, attrition $(10.4 \%)$, mobility $(5.8 \%)$, broken teeth (5.6\%) and alveolar abscess (6.3\%). Considering the pattern of inflammation, $43.5 \%$ had inflammatory oro-dental problems and $56.5 \%$ had non-inflammatory oro-dental problems. Examination of the teeth revealed that only $1.4 \%$ had no affected teeth, $30.4 \%$ had two affected teeth followed by one tooth $(24.2 \%), 15.0 \%$ four and $11.6 \%$ had three, but $17.4 \%$ had all the teeth were affected. It was also found that $39.4 \%$ of the affected teeth were in the maxillary region and $38.4 \%$ in the mandibular region and $20.8 \%$ had both the region were affected. About two third of the respondents had no missing teeth (66.4\%), $13.5 \%$ had two, $11.4 \%$ had one missing tooth. Regarding the number of filling teeth, $76.3 \%$ had no filling teeth. One tenth $(10.1 \%)$ had one filling tooth and $7.7 \%$ had one filling tooth. On 
examination also revealed that $31.9 \%$ had unhealthy gums, $3.9 \%$ had unhealthy tonsils, and $10.3 \%$ had diseased tongue. $10.7 \%$ had diseases mucosa and $4.1 \%$ had apthus ulcer. disease was higher among the patients having habituated with chewing areca nut and lime $(35.0 \%)$ than non-inflammatory disease $(26.1 \%)$.

Table 1: Relationship between oro-dental problems and socio-demographic characteristics

\begin{tabular}{|c|c|c|}
\hline Sociodemographic characteristics & Frequency & Percent \\
\hline \multicolumn{3}{|l|}{ Age in years } \\
\hline $15-24$ & 123 & 29.7 \\
\hline $25-34$ & 145 & 35.0 \\
\hline $35-44$ & 82 & 19.8 \\
\hline $45+$ & 64 & 15.5 \\
\hline \multicolumn{3}{|c|}{ Mean \pm SD $(\mathrm{yrs})=31.5 \pm 11.4$ years; range $=15-79$} \\
\hline \multicolumn{3}{|l|}{ Sex } \\
\hline Male & 276 & 66.7 \\
\hline Female & 138 & 33.3 \\
\hline \multicolumn{3}{|l|}{ Education of the respondents } \\
\hline Illiterate & 53 & 12.8 \\
\hline Primary & 65 & 15.7 \\
\hline Secondary' & 54 & 13.0 \\
\hline s.s.c & 62 & 15.0 \\
\hline H.S.C & 93 & 22.5 \\
\hline Degree $(+)$ & 87 & 21.0 \\
\hline
\end{tabular}

\section{Relationship between food habit of the patients and} oro-dental problems

Analysis indicated that no statistically significant association was found between the oro-dental problems and food habit except liking of sweet and habit of areca nut and lime. This indicated that the

\section{Knowledge and care seeking behavior of oro-dental problems}

It was observed that $55.3 \%$ of the patients had knowledge on causes of dental diseases and the $79.9 \%$ among the reportedly mentioned that it is due to irregular cleaning of teeth followed by taking

Fig 1: Percentage distribution of patients by level of education and oral dental problems

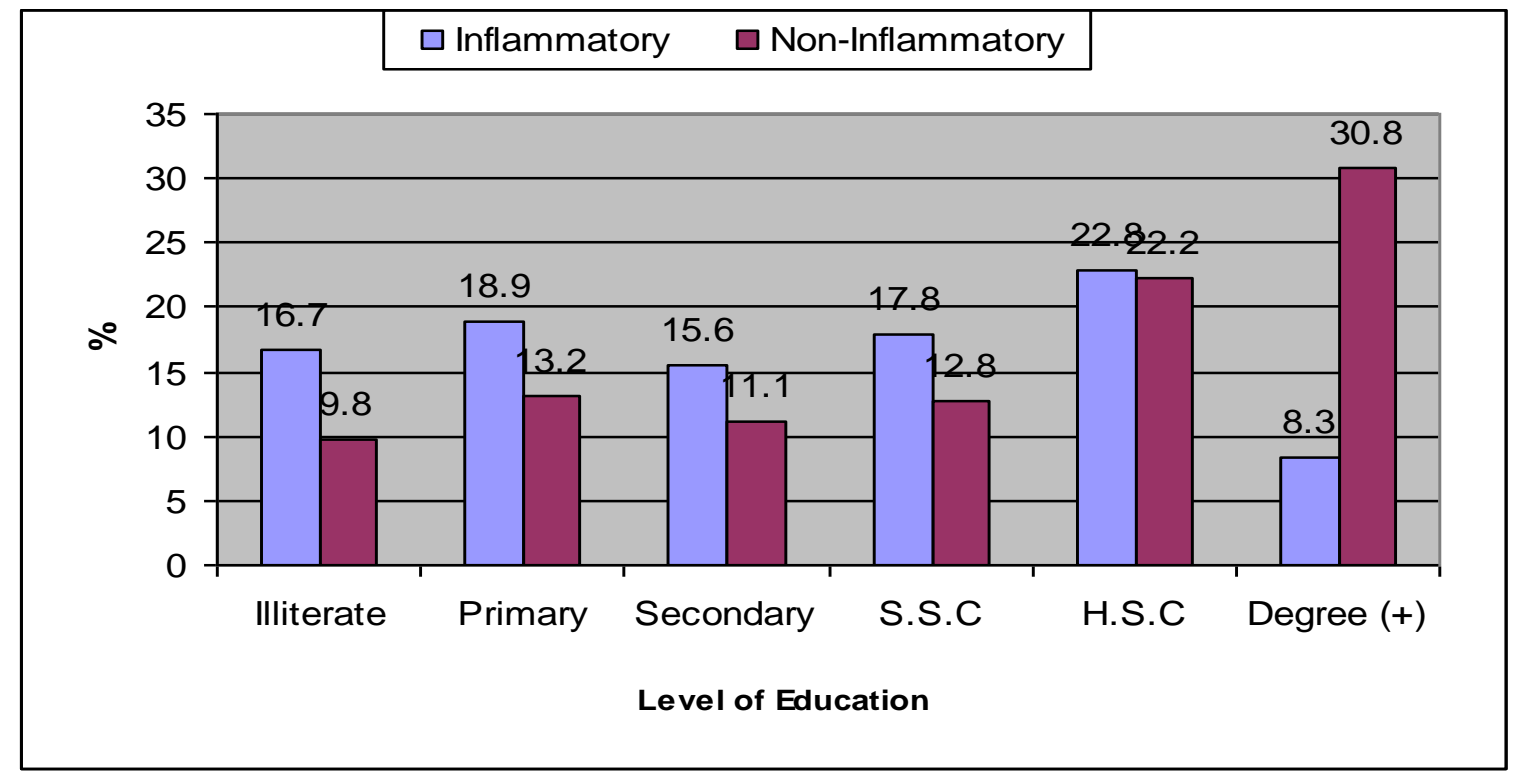

proportion of no inflammatory dental problems were higher among the patients habituate with sweet $(82.5 \%)$ compared to inflammatory disease $(72.2 \%)$. But the proportion of inflammatory excessive sweets $(11.8 \%)$. It was found that only one tenth of the patients visited to dental surgeon for their dental problems. 
For bi-variate analysis, the oro-dental problems were classified into two groups such as inflammatory and non-inflammatory problems. Out of 414 patients, $43.5 \%$ had inflammatory oro-dental problems and education was higher in non-inflammatory disease $(0.9 \%)$ than inflammatory disease $(3.9 \%)$. Similar to fathers, the mothers level of education indicating that the illiterate mothers were higher among the patients

Table 2: Distribution of patients by oro-dental problems and food habit

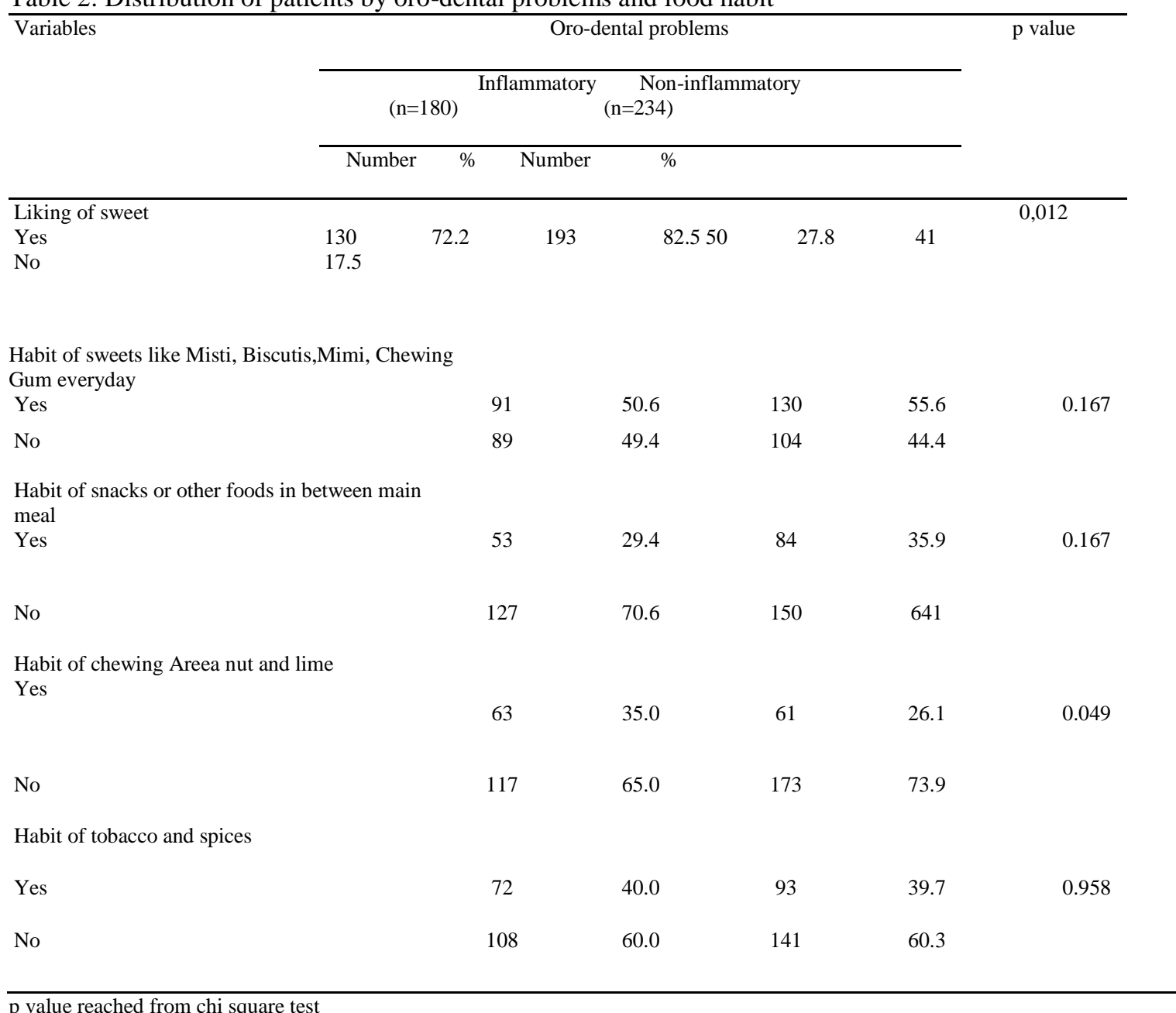

$56.5 \%$ had non-inflammatory oro-dental problems. The mean age of the patients with inflammatory disease was $31.6 \pm 11.9$ years, but the difference was not statistically significant. However, a sex difference was between two groups of patients' indicating the proportion of no inflammatory disease was higher among the male patients $(73.9 \%)$ whereas the proportion of inflammatory disease was higher among the female patients (42.8\%). Similarly, a statistically significant difference was found between two groups of patients' in terms of level of education of the respondents, fathers and mothers. This indicated that the proportion of illiterate patients were higher among the inflammatory disease $(16.7 \%)$ compared to non-inflammatory disease $(9.8 \%)$ whereas respondents having graduate level of education were higher among the non-inflammatory disease $(8.3 \%)$ compared to inflammatory diseases $(8.3 \%)$. Similar pattern of education was also found among the fathers indicating that the proportion of illiterate patients were higher in inflammatory' disease $(45.0 \%)$ compared to non-inflammatory disease $(29.5 \%)$ whereas the proportion of SSC level with inflammatory disease $(61.7 \%)$ than noninflammatory disease $(48.3 \%)$.

\section{Dental care practices}

Regarding the dental care practices, $69.8 \%$ respondents cleaned their teeth once in a day, $29.5 \%$ two times and only $0.7 \%$ cleaned their teeth three times in a day. The most frequent time of cleaning their teeth was before breakfast $(70.8 \%)$ followed by after breakfast and before bed time $(12.3 \%)$, before breakfast and before bed time $(9.7 \%)$, before going to bed $(3.4 \%)$. More than half of the respondents use to clean their teeth by tooth brush and tooth paste (53.6\%) followed by tooth brush and tooth powder $(27.8 \%)$, finger and charcoal $(9.7 \%)$ and finger and tooth powder $(8.9 \%)$. Regarding the methods of cleaning teeth, more than half of the respondents cleaned their teeth from side to side movement (51.2\%) followed by above wards \& lower teeth from down to $(47.1 \%)$ and along the gum line and brush each tooth $(1.7 \%)$. Duration of brushing in each session indicated that highest percentage of respondents brushed teeth more than five minutes $(37.9 \%)$ followed by two minutes $(28.7 \%)$, five minutes $(17.6 \%)$ etc. It was also found that only 
$12.6 \%$ of the respondents had habit of cleaning teeth after taking food or sweet.

\section{Food habit of the patients}

It was observed that $78.0 \%$ of the patients had liking of sweets followed by $53.4 \%$ had habit of sweets like Misti, Biscutis, Mimi, Chewing gum everyday, $33.1 \%$ had habit of snacks or other foods in between main meal, $39.9 \%$ had habit of tobacco and spices and $30.0 \%$ had habit of chewing area nut and lime. problems among the patients coming to a hospital which may be preventable. In this study it was observed that highest percentage of the patients had complaints of periodontitis $(73.9 \%)$ followed by gingivitis $(64.3 \%)$, dental caries $(40.6 \%)$ attrition $(10.4 \%)$, mobility $(5.8 \%)$, broken teeth $(5.6 \%)$ and alveolar abscess $(6.3 \%)$. Considering the pattern of inflammation, $43.5 \%$ had inflammatory oro-dental problems and $56.5 \%$ had non-inflammatory orodental problems. Examination of the teeth revealed

Table 3: Distribution of patients by oro-dental problems and knowledge and dental care seeking behavior

\begin{tabular}{|c|c|c|c|c|c|}
\hline \multirow[t]{3}{*}{ Variables } & \multicolumn{2}{|c|}{ Oro-dental } & \multicolumn{2}{|c|}{ problems } & $\mathrm{p}$ value \\
\hline & \multicolumn{2}{|c|}{$\begin{array}{l}\text { Inflammatory } \\
\quad(\mathrm{n}=180)\end{array}$} & \multicolumn{2}{|c|}{$\begin{array}{l}\text { Non-inflammatory } \\
(n=234)\end{array}$} & \\
\hline & Number & $\%$ & Number & $\%$ & \\
\hline \multicolumn{6}{|c|}{$\begin{array}{l}\text { Knowledge on causes of dental disease } \\
\text { Yes }\end{array}$} \\
\hline & 84 & 46.7 & 145 & 62.0 & 0.002 \\
\hline No & 96 & 53.3 & 89 & 38,0 & \\
\hline \multicolumn{6}{|c|}{$\begin{array}{l}\text { Visit dental surgeon for dental problems } \\
\text { Yes }\end{array}$} \\
\hline & 8 & 4.4 & 40 & 17.1 & 0.001 \\
\hline No & 172 & 95.6 & 194 & 82.9 & \\
\hline
\end{tabular}

\section{Relationship between Knowledge and care seeking behavior and oro-dental problems}

Bi-variate analysis revealed that the knowledge of causes of dental problems higher among the patients having non-inflammatory disease $(62.0 \%)$ compared to inflammatory disease $(46.7 \%)$ and the difference was statistically significant. Similarly, the proportion of visiting dental surgeon was higher among the patients having non-inflammatory disease $(17.1 \%)$ than the inflammatory disease $(4.4 \%)$ and the difference was statistically significant.

Relationship between Knowledge and care seeking behavior and oro-dental problems

$\mathrm{Bi}$-variate analysis revealed that the knowledge of causes of dental problems higher among the patients having non-inflammatory disease $(62.0 \%)$ compared to inflammatory disease $(46.7 \%)$ and the difference was statistically significant. Similarly, the proportion of visiting dental surgeon was higher among the patients having non-inflammatory disease $(17.1 \%)$ than the inflammatory disease $(4.4 \%)$ and the difference was statistically significant.

\section{DISCUSSION}

The study on prototype of oro-dental problems was carried out among the 414 respondents in the outpatient department of Ohud and implant center, Bogra inspective of age and sex. Most of the studies on dental diseases conducted nationally and internationally emphasized in periodonties dental caries and gingivitis only. In this study initiation has been taken to find out the prototype of oro-dental that only $1.4 \%$ had no affected teeth, $30.4 \%$ had two affected teeth followed by one tooth $(24.2 \%), 15.0 \%$ four and $11.6 \%$ had three, but $17.4 \%$ had all the teeth were affected.

The mean age of the patients with inflammatory disease was $31.6 \pm 10.8$ years and that of noninflammatory disease was $31.5 \pm 11.9$ years, but the difference was not statistically significant. However, a sex difference was between two groups of patient's, indicating the proportion of noninflammatory disease was higher among the male patients $(73.9 \%)$ whereas the proportion of inflammatory disease was higher among the female patients $(42.8 \%)$. Regarding the father's occupation, the proportion of agriculture worker were higher in inflammatory disease (28.9\%) compared to noninflammatory disease $(15.0 \%)$, but the proportion of businessmen were higher in non-inflammatory disease $(34.6 \%)$ than inflammatory disease $(23.9 \%)$ and the difference was statistically significant. On the contrary, the mother's occupation indicating that the proportion 01 housewives were higher in inflammatory disease $(95.0 \%)$ than no inflammatory disease $(88.0 \%)$ and the service holders were higher in non-inflammatory disease $(11.1 \%)$ than inflammatory disease $(4.4 \%)$ and the difference were statistically significant.

To this the proportion of one time tooth cleaning was higher among the patients with inflammatory oro-dental problems than non-inflammatory diseases $(62.8 \%)$. Similarly, a statistically significant difference was found in terms of timing of tooth cleaning indicating the proportion of patients 
habituated to cleaning tooth brushing before breakfast was higher among the inflammatory diseases $(86.7 \%)$ than non-inflammatory diseases $(58.5 \%)$. However, no statistically significant difference was found between two groups of patients in terms of materials and instruments used for cleaning the teeth though the proportion of tooth brush and tooth paste/powder were higher among the patients with non-inflammatory diseases than inflammatory diseases. But statistically significant association was found with dental problems and methods of cleaning teeth indicating the proportion of correct method of cleaning of teeth was higher among the patients with non-inflammatory diseases $(53.0 \%)$ than inflammatory disease $(39.4 \%)$.

So this study shown that dental disease one less among the respondents who brush their teeth other breakfast than those who brush their teeth before breakfast. In this study indicated that the proportions of non-inflammatory dental problems were higher among the patients habituate with sweet $(82.5 \%)$ compared to inflammatory disease $(72.2 \%)$. But the proportion of inflammatory disease was higher among the patients having habituated with chewing areca nut and lime $(35.0 \%)$ than non-inflammatory disease $(26.1 \%)$. This finding is similar to the finding of others [7] is findings is also consistent to other researchers [8], who conducted a comprehensive study in the department of pediatric dentistry, University of many In this study knowledge of causes of dental problems higher among the patients having non-inflammatory disease $(62.0 \%)$ compared to inflammatory disease $(46.7 \%)$ and the difference was statistically significant. Similarly, the proportion of visiting dental surgeon was higher among the patients having noninflammatory disease $(17.1 \%)$ than the inflammatory disease $(4.4 \%)$ and was corresponds to the statement [6]. They state that knowledge about oral hygiene is an important aspect of our daily life, which play a great role in the prevention of dental caries and intensification of oral hygiene constitutes the best prophylaxis of oral-dental diseases. Similar result was also found in another study [10] which shows $65.00 \%$ of lower caries experience in four years of aged children whose mothers received preventive education program regarding dental caries 4 years ago than in children whose mother did not receive counseling.

\section{CONCLUSION}

The results shows the prevalence of periodontitis in the community is $73.9 \%$ followed by gingivitis $(64.3 \%)$ dental caries (40.6\%) attrition (10.4\%) mobility $(5.8 \%)$ broken teeth $(5.6 \%)$ and alvedor abscess $(6.3 \%)$. So we can come to conclusion that periodontitis, gingivitis and dental caries in are in important public health problem in Bangladesh. A significant association was found between the orodental problems and food habit except liking of sweet and habit of area nut and lime. Also the proportion of non-inflammatory dental problems was higher among the patients habituate with sweet compared to inflammatory disease. But the proportion of inflammatory disease was higher among the patients having habituated with chewing areca nut line than non-inflammatory disease. Knowledge of the causes of dental problems was higher among the patients having non-inflammatory disease compared to inflammatory disease. Similarly, the proportion of visiting dental surgeon was higher among the patients having non-inflammatory disease. These common diseases are multifactorial diseases and the factors responsible for these diseases are preventable by various awareness programs, regular practice of of oral hygiene and visiting dental surgeon in a regular basis.

\section{REFERENCES}

1. Anwarul A, Bachchu MA, 1989. Prevention of Dental caries and the science of nutrition. Bangladesh Dental Journal. 6(1): 5-9.

2. Awal MA, Shahidullah, 1988. Knowledge of mothers about dental caries and its prevention in a selected urban area. Journal of preventive and social medicine. 16(1): 710.

3. Alam MR, Sarkar AM, 1988. Prevalence of dental caries of rural primary school children. Bangladesh dental society journal. 1(1): 14-17.

4. Haque A, 1995. Oral Health. In Rashid K.M. Khabiruddin and Hyder S. Text book of community medicine and public health, second edition, RKH publishers, Bogra. 334-338.

5. Haque MJ, 1992. A study on prevalence of caries teeth in preschool children in a selected upazila in Bangladesh. Disertation of NIPSOM, 1991-92.

6. Begum A, 1982. oral health education in Bangladesh. Bangladesh dental journal. 2(1): 25-27.

7. Rashid A, 1994. Caries prevalence in preschool children of high and low sugar consumption groups. Bangladesh Dental Journal. 10(1): 37-38.

8. Tinanaff N, Palmer CA, 2000. Dietary determinate of dental caries and dietary recommendations for preschool children. Dental public health. 60(3): 197-206.

9. Lange DE, 1988. The Practical approach to Improve Oral Hygiene. Internal Dental Journal. 38(2); 154-160.

10. Nizel AE, 1980. Nutrition on preventive dentistry, science and practice, Philadelphia, Sauderas, intemational Dental Journal. 43(5): 120-126. 
